\title{
Bottom-up Modeling of Local Energy Markets within a Pan- European Wholesale Electricity Market Model
}

This paper was downloaded from TechRxiv (https://www.techrxiv.org).

\section{LICENSE}

CC BY-NC-SA 4.0

SUBMISSION DATE / POSTED DATE

$12-03-2020$ / 14-03-2020

\section{CITATION}

Schmitt, Carlo; Samaan, Kenneth; Schwaeppe, Henrik; Moser, Albert (2020): Bottom-up Modeling of Local Energy Markets within a Pan-European Wholesale Electricity Market Model. TechRxiv. Preprint. https://doi.org/10.36227/techrxiv.11974161.v1

$\mathrm{DOI}$ 


\title{
Bottom-up Modeling of Local Energy Markets within a Pan-European Wholesale Electricity Market Model
}

\author{
Carlo Schmitt, Kenneth Samaan, Henrik Schwaeppe, Albert Moser \\ Institute for High Voltage Equipment and Grids, Digitalization and Energy Economics (IAEW) \\ RWTH Aachen University \\ Aachen, Germany \\ c.schmitt@iaew.rwth-aachen.de
}

\begin{abstract}
The energy system decarbonization and decentralization require coordination schemes for distributed generators and flexibilities. One coordination approach is local energy markets for trading energy among local producers and consumers. The resulting local coordination leads to the questions of how the interaction between local and wholesale markets will be designed and of how the introduction of local energy markets influences the wholesale market system. Therefore, this paper proposes a bottom-up modeling method for local markets within a panEuropean wholesale market model. Furthermore, an aggregationdisaggregation method for local markets is developed to reduce computational effort. A case study for local markets in Germany shows the computational advantages of the aggregationdisaggregation method. Preliminary results indicate the impact of different interaction designs between local and wholesale markets on the wholesale market and show the need for further research.
\end{abstract}

Index Terms-Local Energy Market, Wholesale Electricity Market, Lagrangian Relaxation

\section{INTRODUCTION}

The ongoing expansion of energy sources and flexibilities such as photovoltaic (PV) power plants, battery storage systems, and power-to-heat technologies on the distribution grid level bears the need for efficient coordination of these technologies. Such distributed technologies are conventionally coordinated using aggregators such as utilities and virtual power plants (VPPs) that aggregate distributed energy supply, demand and flexibility and procure them on the wholesale market (WSM). One alternative coordination approach to allocating energy on the local level is via local energy markets (LEMs) where local market participants can trade energy directly with one another [1]. This reduces the dependence on aggregators for trading local participants' energy at the WSM and further leads to the question of how the introduction of LEMs influences the trading volumes and prices in the WSM. Additionally, LEMs are still innovative approaches.

(C)2020 IEEE. Personal use of this material is permitted. Permission from IEEE must be obtained for all other uses, in any current or future media, including reprinting/republishing this material for advertising or promotional purposes, creating new collective works, for resale or redistribution to servers or lists, or reuse of any copyrighted component of this work in other works.
Interactions and interfaces between local and wholesale (centralized) market systems require further investigation and are still undetermined; e.g. whether trading is limited to locally produced energy or also trading of WSM energy on local markets by WSM agents is still open to (regulatory) discussion [2]. The different interfaces have a high impact on local market prices and volumes and accordingly on the WSM. Surveys have further shown that part of residential customers show a higher willingness to pay for regional energy, whereas other customers use LEMs for economic optimization [3], [4]. Therefore, different forms of LEM designs and regulations are possible and need to be considered to estimate the impact of LEMs on WSMs. In [5], a co-simulation of LEMs and a WSM is described, although only for one specific interface design of LEMs and only for a small-scale WSM model. In most system studies, e.g. in [6], the local coordination of distributed energy resources and its impact on the European wholesale market modeled as a preprocessing step, but no optimization of distributed flexibilities on the wholesale market is considered. In [7], distributed technologies are aggregated as local VPPs on a high-voltage/ medium-voltage (HV/MV) substation level and optimized within a pan-European wholesale market model. However, to the best of our knowledge, there exists no framework to model the different design forms of LEMs within the pan-European wholesale electricity market. Furthermore, considerations of distributed flexibilities within wholesale market models so far rely on aggregating methods or only a subset of the flexibilities and do not allow for a detailed dispatch on a local level.

This paper introduces a framework for modeling LEMs within a pan-European electricity market model. Our model further allows for dispatching of millions of distributed flexibilities on an individual building or business level. The framework is based on both a multi-modal bottom-up modeling approach to distributed energy technologies as well as electrical and thermal demands [8] and the multi-modal market optimization with explicit consideration of these distributed energy sources within a pan-European Lagrangian relaxation [7], [9]. For the framework presented in this paper, we extend the multi-modal 
Lagrangian relaxation approach to model LEMs. Our model is further designed to consider the different WSM interface options and their impact on the wholesale market. The rest of the paper is organized as follows. In section II, the modeling of local market participants is introduced and the local market optimization model is further described in detail. Section III describes the wholesale electricity market model as well as the integration of LEM models. Furthermore, it describes an aggregation-disaggregation method for ensuring the dispatch at the individual building level of LEM participants within the iterative Lagrangian relaxation. Section IV presents an example case study considering LEMs in Germany as well as the pan-European Electricity market. The results are discussed in section $\mathrm{V}$, followed by a conclusion in the final section.

\section{LocAl EnERGy MARKet Model}

To optimize LEMs with high spatial and temporal granularity, the market participants are modeled on a single building or business level. Based on the bottom-up data described in [8], market participants are differentiated as household buildings (HH) and commerce, trade \& services (CTS) or industry (IND) businesses. The data comprises over twenty million buildings and businesses on a sub-postal code level [10]. Each building or business has an assigned hourly thermal and electrical load profile as well as heat generation technologies. Furthermore, roof PV and battery systems are assigned to individual units. Every market participant thus represents a multi-modal energy management system (EMS) (cf. Figure 1). We define the LEM areas in this paper as the areas supplied by HV/MV substations because market participants are electrically connected by the corresponding medium voltage distribution grid. Our modeling approach is nonetheless not restricted to this area definition and can be parametrized dynamically for different LEM area configurations.

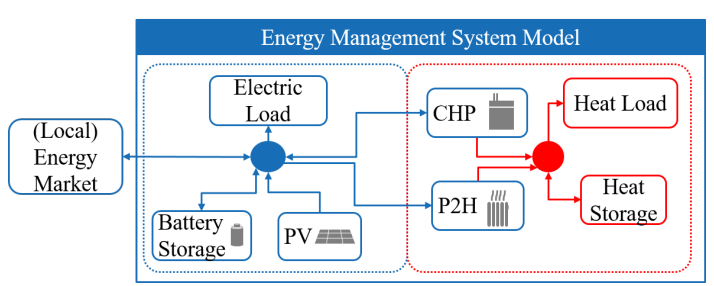

Fig. 1. Exemplary EMS Model of Local Market Participant.

\section{A. Interaction of LEMs and Wholesale Markets}

Because the interactions and interfaces between local and central markets are still undetermined, different options for interfaces need to be considered when modeling LEMs. In [2], three different options are discussed. The first one focuses on local market participation restricted to local prosumers and technologies. This means that residual energy not sold or bought on the LEM needs to be procured by aggregators in the WSM. The other two options concern a new role for those WSM aggregators. They either have the opportunity to directly operating the local market platform and neutrally

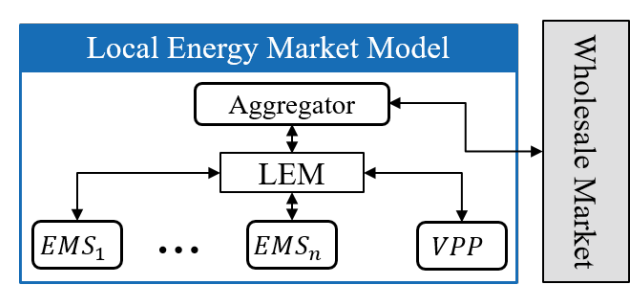

Fig. 2. LEM Model Structure Overview.

offering energy at (expected) wholesale market clearing prices (MCPs). Alternatively, the LEM is operated by a market operator and aggregators compete in the local market and have the opportunity to trade between the LEM and the WSM. Due to the same marginal cost (the corresponding wholesale MCPs) for WSM aggregators and thus no significant market power on the local level, both options are assumed to yield the same local market results (cf. [2]) and can be treated equally from a modeling perspective. The two options considered in this paper for modeling the interplay between local and wholesale markets are as follows: (1.) the indirect interface option with LEM trading restricted to local assets and (2.) the interface option with wholesale agents trading on LEMs.

The first interface option can be modeled by offering constant prices on the LEM platform, assuming backup prices for selling and buying energy from aggregators to be higher (or lower) than the wholesale spot prices due to mark-ups induced by the backup aggregators. This simplification is based on the fact that backup instances already define the marginal cost and earnings for buying and selling energy for each market participant and therefore already act indirectly as the price-setting instance on the LEM. If the different local market participants have differentiated backup prices, the energy bought to and sold from backup aggregators can alternatively be modeled within the respective EMS models. This interface option corresponds to the maximization of local energy consumption because it is not sensitive to the wholesale market price and the local MCP is advantageous in comparison to the backup price for both local buyers and sellers of energy. The local optimization is independent of the wholesale MCP and the resulting residual load time series can be considered as part of the static load within the corresponding market zone within the wholesale market model. The second, wholesale marketbased interface option, where aggregators pass the wholesale market prices on to the local market, does incentivize changed bidding and prices on the LEM. Local trading now depends on not only local production and consumption but also on the (expected) prices on the wholesale market. The residual energy of the LEMs thus differs for this interface in comparison to the first interface option. Assuming ideal competition, a single aggregator with no price-making decisions can approximate the set of wholesale market aggregators. Therefore, a single aggregator model for trading between local and wholesale markets is used to trade the local residual energy demand on the wholesale market (cf. Fig.2). 
Equal aggregator price bids for buying and selling energy at wholesale market prices incentivize no local trading but only the optimization in the wholesale market for distributed EMS. Local trading thus requires a price offset between buying energy from and selling energy to the wholesale system. That offset can, on the one hand, be induced by regulatory measures to incentivize local trading such as the remittance of transmission grid charges for locally traded energy. On the other hand, it can be induced by the higher willingness to pay for local energy by local prosumers [3], [4]. The higher this offset, the more similar the results for maximized local matching and a direct interface between LEMs and WSMs are. A maximized matching of local energy leads to a smoothed residual load profile whereas the introduction of the external price signal of the wholesale market incentivizes a more WSM-dependent optimization. In contrast to this paper, [5] assumes that if LEMs are considered, local generators have access to both local and wholesale markets. Since the second interface option in this paper is modeled by a neutral interface between markets, the wholesale MCP is passed to the LEM through the aggregator. The local EMS are thus indirectly trading at the wholesale market through the aggregator and this modeling approach generates the same dispatch results for the EMS as the trading option for EMS in [5].

\section{B. Local Energy Market Model Formulation}

The different market design options for LEMs (cf. [11]) are approximated in this paper with a linear economic dispatch model for local energy. The model minimizes the cost for local load coverage (1) subject to the local energy balance (2) and constraints of the market participants (3)-(5):

$$
\min \sum_{e \in E M S} c_{e}^{T} \boldsymbol{x}_{e}+\sum_{v \in V P P} c_{v}^{T} \boldsymbol{x}_{v}+c_{+}^{T} \boldsymbol{p}_{+}^{L E M}+c_{-}^{T} \boldsymbol{p}_{-}^{L E M}
$$

s.t.

$$
\begin{aligned}
\sum_{e \in E M S} \boldsymbol{x}_{e}^{L E M}+ & \sum_{v \in V P P} \boldsymbol{x}_{v}^{L E M}+\boldsymbol{p}_{+}^{L E M}-\boldsymbol{p}_{-}^{L E M}=0, \forall t \\
& A_{e} \cdot \boldsymbol{x}_{e} \leq b_{e}, \forall e \in E M S \\
& A_{v} \cdot \boldsymbol{x}_{v} \leq b_{v}, \forall v \in V P P \\
\boldsymbol{p}_{+}^{L E M} & \geq 0, \boldsymbol{p}_{-}^{L E M} \geq 0
\end{aligned}
$$

The dual variable $\pi_{t}$ of constraint (2) corresponds to the MCP of the LEM model. When considering yearly time horizons for LEM optimization, a rolling optimization is applied to reduce model complexity while still taking into account time-coupling constraints. The modeling of the EMS in (3) is based on [7] and the modeling of the VPPs (4) for local renewables, e.g. non-building PV plants and wind power plants, is described in [12]. The objective function coefficients $c_{-}$and $c_{+}$for the EMS and VPPs cover individual costs for generation of local participants and can be expanded to include regulatory aspects, e.g. taxes [7]. The variables $\boldsymbol{p}_{+}$and $\boldsymbol{p}_{-}$account for the positive and negative residual energy of the local system. This residual energy is traded through the aggregator at the
WSM. The prices $c_{-}$and $c_{+}$thus correspond to the prices for selling to and buying from the wholesale system. If the local energy matching is maximized, $c_{-}$and $c_{+}$are assumed constant with $0<c_{-}<<c+$. For the direct interface option, the price for selling to the wholesale market $c_{-}$is equal to the wholesale MCP $\lambda$ and the price for buying from the WSM is $c_{+}=\lambda+\Delta_{c}$. The price offset $\Delta_{c}$ describes the incentive for local trading, either as a regulatory component (for example, the remittance of transmission grid charges) or the higher willingness to pay for local energy (cf. section II.A). If $\Delta_{c}$ is equal to zero, there is no incentive for LEM trading and the model optimizes the dispatch of the distributed EMS at the WSM with no consideration for local trading.

\section{Aggregation of EMS and Decomposition of LEMs}

The LEM formulation (1)-(5) allows for linear modeling of LEMs. To account for a high number of market participants, the model complexity can be reduced by aggregating sets of market participants. For this, market participants and their technology portfolios within an LEM are aggregated on the basis of spatial and sectoral criteria, i.e. all HH, IND and CTS buildings within a spatial area are aggregated as one EMS for each sector. Within each sector aggregation, heat loads are aggregated for each heating technology. Thus, every aggregated EMS consists of several heat management systems that are only coupled electrically (cf. [8]). The definition of aggregation levels is highly flexible; for example, market participants can be aggregated on the basis of their postal code area. The LEM model is then optimized for fewer, aggregated participants and thus fewer variables and constraints. However, by using aggregation, the constraints of individual market participants might be violated. Examples of aggregation errors include heat storage capacities and battery storage dis- and charging capacities.

To correct for the aggregation errors, the LEM is further decomposed into aggregation areas (which can be seen as subLEMs) and for each area, a reduced LEM model is solved for only the subsets $\overline{E M S}$ and $\overline{V P P}$ of the market participants in the corresponding aggregation area. The objective function of the decomposed economic dispatch is given in (6):

$$
\min \sum_{e \in \overline{E M S}} c_{e}^{T} x_{e}+\sum_{v \in \overline{V P P}} c_{v}^{T} x_{v}+\hat{c}_{+}^{T} \hat{\boldsymbol{p}}_{+}^{L E M}+\hat{c}_{-}^{T} \hat{\boldsymbol{p}}_{-}^{L E M}
$$

In contrast to (1), the residual energy is priced with the MCP of the aggregated LEM model, i.e. $\hat{c}_{+}=\hat{c}_{-}=\pi$, to reflect for overdemand or undersupply in the total LEM area. The decomposed model constraints are structured as (2)-(5), but only with consideration for the subsets $\overline{E M S}$ and $\overline{V P P}$. After solving each LEM of each aggregation area, the residual energies $\hat{\boldsymbol{p}}_{-}$and $\hat{\boldsymbol{p}}_{+}$are aggregated to calculate the total residual energy $\boldsymbol{r}=\boldsymbol{p}_{+}-\boldsymbol{p}_{-}=\sum \hat{\boldsymbol{p}}_{+}-\hat{\boldsymbol{p}}_{-}$of the LEM. 


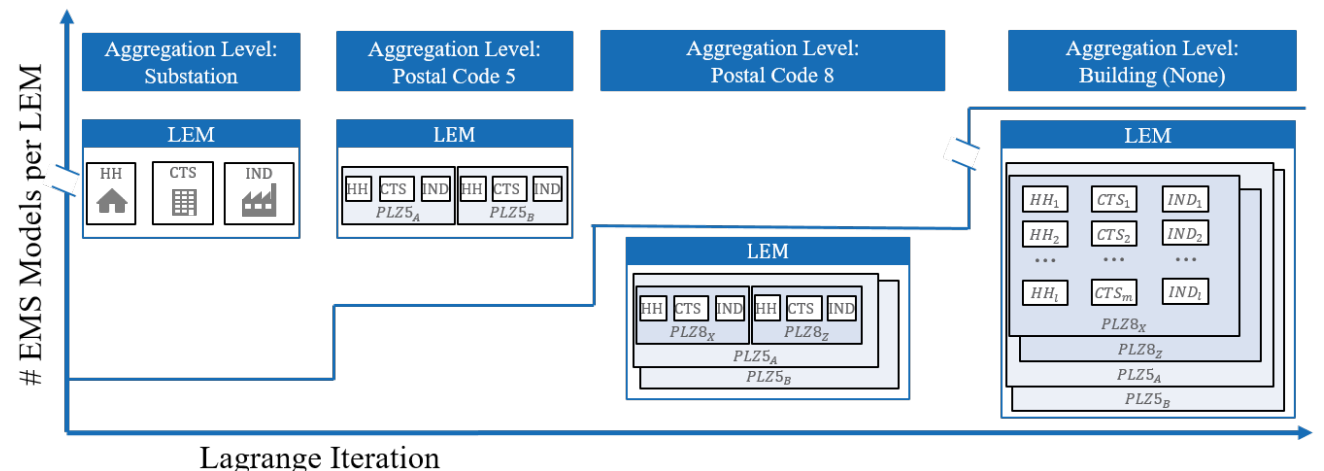

Fig. 3. Aggregation-Disaggregation Method.

\section{INTEGRATED WhOLESALE AND LOCAL ENERGY MARKET MODEL}

The pan-European market model is a fundamental energy system model based on unit commitment decisions. It comprises the modeling of power plants, renewable plants, largescale (hydro) storage systems as well as the electrical load for every country considered within the market optimization. The optimization model is decomposed with a Lagrangian relaxation of the load balancing constraint for each market area [9]. Within each iteration, the Lagrangian price coordinators (the MCPs of the different market zones) are passed to the submodels for power plants, renewables, and storages that optimize their dispatch according to the currently offered MCP. A market coupling is carried out subsequently and the Lagrangian coordinators for each market zone are adjusted according to the market coupling results [9]. By applying detailed bottom-up modeling of distributed energy structures [8], the distributed flexibility can further be considered within the European market model. The (aggregated) market-based optimization of distributed flexibilities within the Lagrangian relaxation process is introduced in [7] (and [9]), where the distributed technologies are aggregated on the HV/MV substation level. This approach is extended to account for LEMs as described in section II. When considering the LEM configuration with maximized local matching and no direct interface to the wholesale markets, the local optimization is independent of the MCPs and can thus be optimized before considering the WSM model. The resulting load of the local areas is then considered within the market model as a static load. Local surplus generation of renewables is considered as renewables bidding in the market. If otherwise the interface option with direct trading between LEMs and the WSM is considered, the individual LEM models (1)-(5) are optimized within each Lagrangian iteration similar to the other submodels for storages, power plants, etc.

\section{A. Aggregation-Disaggregation Method}

The Lagrangian relaxation model [9] already allows for parallelized optimization of ten- to hundreds of thousands of distributed flexibilities, given enough computing resources. To reduce the computation time of the market model further,

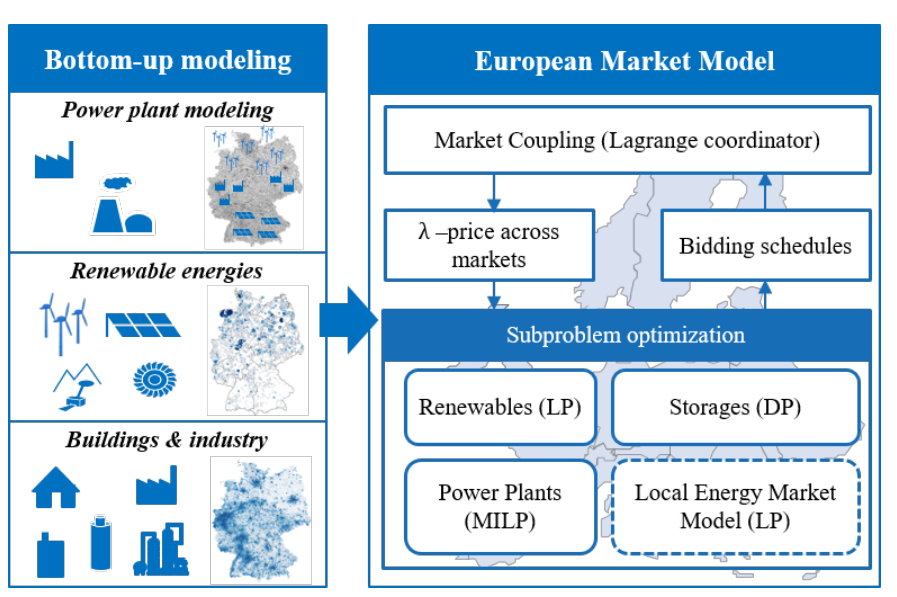

Fig. 4. European Market Model Overview.

we develop an aggregation-disaggregation method for the LEMs within the Lagrangian relaxation approach. This method allows for both solving the integrated market model for local and wholesale markets in reasonable computation time and a dispatch on every building or business EMS level. The distributed generators and loads are aggregated as described in [8] and different aggregation levels are defined. The highest aggregation level here is defined as $\mathrm{HV} / \mathrm{MV}$-substation levels where all buildings and businesses are aggregated as one EMS for each sector (HH, CTS \& IND). More granular aggregation levels are postal and sub-postal code areas, i.e. German postal code 5 (PC5) and Postal code 8 (PC8, cf. [10]) areas. The most granular level is defined as a single building or business within an LEM and thus requires no aggregation. In general, the computational complexity increases with granularity, i.e. the lower the level of aggregation, the higher the computational effort. In contrast, the errors due to aggregation decrease with higher granularity.

The concept of the aggregation-disaggregation method is to vary the levels of aggregation within the Lagrangian relaxation approach. At the beginning of the Lagrangian iterations, the highest aggregation level (substations) is used. In higher iterations, the granularity is increased gradually to reduce 


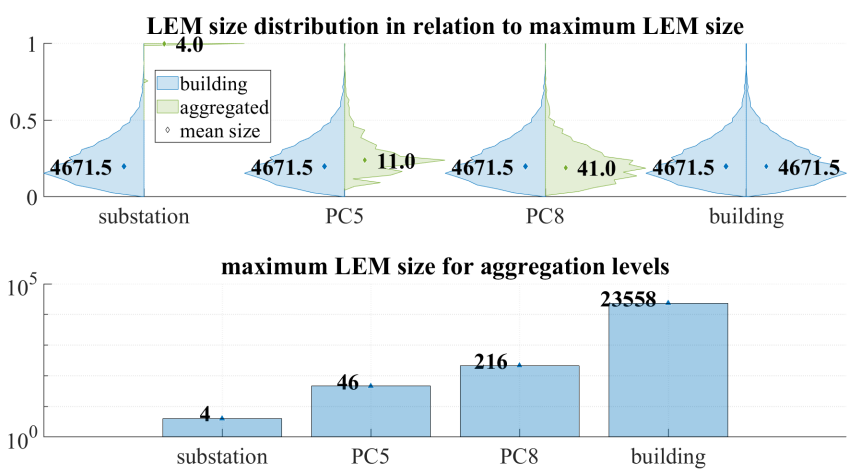

Fig. 5. Size distribution of LEMs.

aggregation errors (cf. Fig. 3). For large LEMs with a high number of participants, the decomposition method described in section II.C can be used for further complexity reduction. We exploit the trade-off between computation time and accuracy to reduce the total computation time and still get accurate results of the EMS in the later iterations. The update process of the Lagrange coordinator is based on total supply and demand bids. In the first iterations, the oversupply or overdemand of bids is mainly driven by large-scale power plants and the baseload within each market zone. The aggregation errors have a comparatively minor influence on the update process in the first iterations.

\section{EXemplary CASE Study}

For the case study, we examine the impact of an introduction of LEMs in Germany on the European day-ahead market by considering a scenario with high penetration of renewables and flexible technologies as well as storages. The scenario is taken from [7]. The LEM configuration based on the HV/MV substations leads to 4330 different LEMs for Germany with more than 22 million individual buildings and businesses. Figure 5 shows an overview of the maximum LEM sizes with respect to the number of market participants as well as the size distribution of LEMs for the different aggregation levels. The upper plot in Figure 5 shows that the more granular the aggregation, the similar the distribution is to the actual size distribution on a building level. The pan-European wholesale market is optimized for both LEM-WSM interface options: A pre-optimized demand time series in Germany based on the maximized local matching represents the first option. The second option is calculated as LEM optimization within the Lagrangian relaxation for two different price parameters. For $\Delta_{c}=0 \mathrm{EUR} / \mathrm{MWh}$, the distributed EMS are directly optimized in the WSM and for $\Delta_{c}=25 \mathrm{EUR} / \mathrm{MWh}$, a regulatory incentive for local trading is examined. The 25 EUR/MWh are derived from forecasts of transmission grid charges in 2030 [13]. The calculations for the example case study are performed on the RWTH computation cluster with 200 parallel jobs for solving the WSM and (aggregated) LEM subproblems with 13 to 15 GB RAM/ 3 cores allocated for each job. When
TABLE I

ITERATION CALCULATION TIMES

\begin{tabular}{|l|l|l|l|l|}
\hline Aggregation Level & Substation & PC5 & PC8 & $\begin{array}{l}\text { Building } \\
\text { (None) }\end{array}$ \\
\hline $\begin{array}{l}\text { approx. Runtime per } \\
\text { Lagrange Iteration } \\
(200 \text { jobs })\end{array}$ & $9 \mathrm{~min}$ & $17 \mathrm{~min}$ & $\mathbf{3 9} \mathrm{min}$ & $\mathbf{1 2 0} \mathrm{h}$ \\
\hline $\begin{array}{l}\text { Use in Iteration } \\
\text { [Start, Stop] }\end{array}$ & {$[1,59]$} & {$[60,79]$} & {$[80,97]$} & {$[98,100]$} \\
\hline
\end{tabular}

optimizing LEMs with building EMS granularity, 1000 parallel jobs are used.

\section{RESUlTs AND Discussion}

The calculation times for each Lagrangian iteration with 200 jobs increase with granularity. Table I contains those resulting calculation times per iteration. Since the calculation time for the different jobs are not deterministic and vary between jobs due to the computing resources' variations, only approximate times can be given. The calculation times on the building level are significantly higher than those at the aggregation levels because a much higher number of market participants per LEM is considered. Nonetheless, the Lagrangian iteration with LEMs on a single building level can be optimized within 24 hours when using 1000 parallel jobs. The total calculation time for 100 iterations when using the aggregation scheme described in the last row of Table I (and using 1000 jobs for the buidling level calculation) thus amounts to approximately 4 days. By using more jobs and/or more computing resources per job, the computation time per iteration can further be reduced. Figure 6 depicts the approximated cumulated computation time for the different aggregation levels when using 200 jobs. An optimization on single EMS level for the considered case of Germany would amount to a calculation time of several hundred days. Even a maximized parallelization, which is limited by the number of LEMs, would only bring this calculation time down to a little more than twenty days. By using the aggregation-disaggregation method developed in this paper, it is possible to calculate the WSM model with integrated LEM optimization within a couple of days while generating a detailed dispatch in the final iterations. One open question that needs to be addressed in future research is the optimal use of aggregation levels. The distribution of LEM sizes for different aggregation (cf. Fig. 5) show that on the PC8 level, approximately 100 distributed EMS are aggregated as one. A more granular aggregation level than PC8 can further increase the level of detail but will also increase computation time. Furthermore, the switch between aggregation levels could be determined based on the Lagrange convergence.

The detailed dispatch of the aggregation-disaggregation method can further be used independently of LEM calculations to evaluate the impact of different energy system scenarios on the distribution grid utilization while taking distributed flexibility participation in the WSM into account. Additionally, when considering taxes on electricity, the result accuracy can 


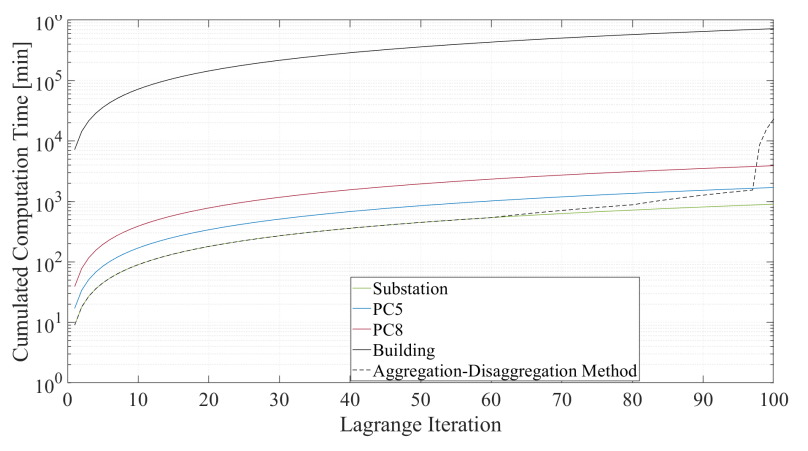

Fig. 6. Cumulated Runtime of different Aggregation Methods within European Market Model.

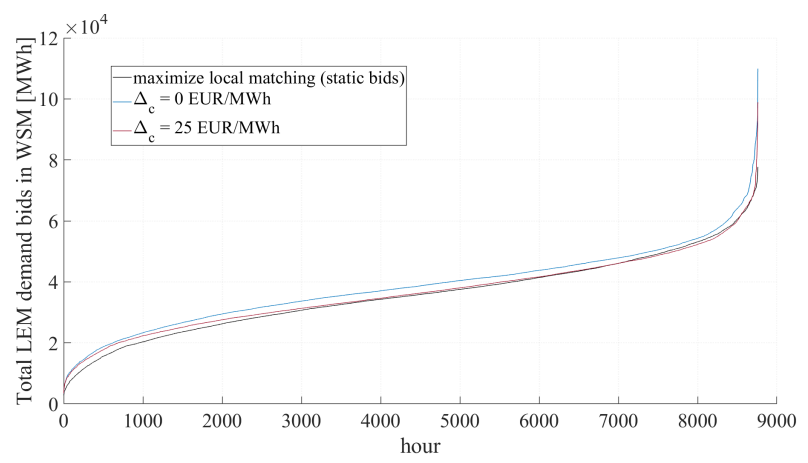

Fig. 7. Annual Load Duration Curve of total LEM demand bids $p_{+}$.

be increased by modeling the electricity exchange between the different EMS in detail.

The results of the market calculation show that even in a scenario with such a high installed capacity of flexible technologies and for maximized local matching of energy, the LEMs are not self-sufficient in all time steps and need to exchange energy with the zonal and pan-European power system. Figure 7 shows the impact of different interfaces on the demand bids in the German market zone. The total demand bids of LEM aggregators (which is equal to the sum of $\boldsymbol{p}_{+}$ for all LEM) are depicted as an annual duration curve for the three LEM scenarios. The resulting demand bids for EMS directly trading at the WSM $\left(\Delta_{c}=0\right)$ are significantly higher than those of the maximized local energy matching. This is based on a surplus of renewable energy generation. A shift of demand thus leads to reduced cost for load coverage. At last, it can be concluded that the introduction of a local trading incentive $\left(\Delta_{c}>0\right)$ shifts the load bids to those of a WSMindependent LEM (max. matching), as discussed in section II. In further studies, the market results from our model can be used to examine the impact of LEMs on the transmission grid by applying power flow and redispatch calculations.

\section{CONCLUSION}

This paper introduces a bottom-up modeling framework for local energy markets within a pan-European wholesale market model. The framework considers different use cases and design options of LEMs and their respective interfaces to the wholesale market system. By introducing an aggregationdisaggregation method within a Lagrangian relaxation of the European market, the model is scalable to model millions of individual local market participants. A case study for LEMs in Germany shows that a granular calculation of millions of buildings in every iteration amounts to hundreds of days of calculation time. The aggregation-disaggregation method presented in this paper can speed-up the calculations while still enabling a granular dispatch in the later iterations. The results show that different interfaces between local and wholesale markets affect the zonal demand and thus need to be considered when designing the interaction of LEMs and wholesale markets. Further studies will focus on the optimal tradeoff between granularity and computation time within the iterations of the Lagrangian relaxation. Subsequently, a detailed analysis of the impact of LEMs on the European wholesale markets as well as transmission grid power flows will be carried out.

\section{ACKNOWLEDGMENT}

Simulations were performed with computing resources granted by RWTH Aachen University under project rwth0487. The authors thank Moritz Nobis and Tom Kulms for the case study data and helpful discussions.

\section{REFERENCES}

[1] E. Mengelkamp, B. Notheisen, C.Beer, D. Dauer, C. Weinhardt, "A blockchain-based smart grid: towards sustainable local energy markets", Computer Science-Research and Development, 33(1-2), 207-214, 2018

[2] C. Schmitt, W. Cramer, M. Vasconcelos, N.Thie, "Impact of spot marke interfaces on local energy market trading", 16th International Conference on the European Energy Market (EEM), 2019

[3] E. Mengelkamp, T. Schönland, J. Huber, C. Weinhardt, "The value of local electricity - A choice experiment among German residential customers", Energy Policy 130, 2019, p. 294-303

[4] N. Günther, L. Fait, E. Groh, H. Wetzel, "Gibt es eine Zahlungsbereitschaft für regionalen Grünstrom?", Energiewirtschaftliche Tagesfragen 69. Jg. (2019) Heft 11

[5] F. Lezama, J. Soares, P. Hernandez-Leal, M. Kaisers, T. Pinto and Z. Vale, "Local Energy Markets: Paving the Path Toward Fully Transactive Energy Systems," in IEEE Transactions on Power Systems, vol. 34, no. 5, pp. 4081-4088, Sept. 2019.

[6] C. Rehtanz, C. Spieker, D. Klein, B. Matthes, and M.-L. Kloubert, "Begleitung Netzentwicklungsplan 2030 (NEMO VI)," 2018.

[7] M. Nobis, T.Kulms, "Evaluating regulatory measures in the German energy transition -A European multimodal market optimization approach including distributed flexibilities", 42nd International Association for Energy Economics (IAEE) Annual Conference, 2019

[8] C. Müller et al., "Modeling framework for planning and operation of multi-modal energy systems in the case of Germany", Applied Energy 250,1132 - 1146, 2019.

[9] S. Raths, "Marktsimulationsverfahren für einen dezentral geprägten Strommarkt", PhD Thesis, RWTH Aachen University, 2020

[10] Digital Data Services (DDS). "Postal code 8 Germany XXL data packages (2014)", Karlsruhe; 2014

[11] W. Cramer et al., "Engaging Prosumers in Local Energy Market Business Models", The 25th international conference and exhibition on electricity distribution (CIRED), 2019

[12] N. Thie, M. Vasconcelos, A.Schnettler, L. Kloibhofer, "Influence of European Market Frameworks on Market Participation and Risk Management of Virtual Power Plants," 2018 15th International Conference on the European Energy Market (EEM), Lodz, 2018, pp. 1-5.

[13] Consentec \& Fraunhofer ISI, "BMWi-Vorhaben Netzentgelte": Auswertung von Referenzstudien und Szenarioanalysen zur zukünftigen Entwicklung der Netzentgelte für Elektrizität”, Study commissioned by the Federal Ministry of Economics and Energy (BMWi), 2018. 